\title{
ON THE INITIAL-BOUNDARY VALUE PROBLEM FOR A BINGHAM FLUID IN A THREE DIMENSIONAL DOMAIN
}

\author{
JONG UHN KIM
}

\begin{abstract}
The initial-boundary value problem associated with the motion of a Bingham fluid is considered. The existence and uniqueness of strong solution is proved under a certain assumption on the data. It is also shown that the solution exists globally in time when the data are small and that the solution converges to a periodic solution if the external force is time-periodic.
\end{abstract}

0. Introduction. The purpose of this paper is to establish the existence of strong solutions to a variational inequality which describes the motion of a Bingham fluid in a bounded three dimensional domain. A Bingham fluid is a rigid viscoplastic fluid which is governed by a special constitutive law such that it moves like a rigid body if a certain function of the stresses does not reach the yield limit and it behaves like a viscous fluid when the yield limit is reached. Since the motion is governed by two entirely different stress-strain relations depending on the state of stresses, the conservation of momentum is expressed in terms of a variational inequality so that one can avoid the difficulty of separating the fluid zone and the rigid zone.

The initial-boundary value problem we shall study is formulated as

$$
\begin{aligned}
\left\langle\frac{\partial u}{\partial t}, w-u\right\rangle+ & a(u, w-u)+b(u, u, w)+J(w)-J(u) \\
& \geq\langle f, w-u\rangle \quad \text { in }(0, T)
\end{aligned}
$$

for each test function $w$ such that $\nabla \cdot w=0$ in $\Omega$ and $w=0$ on $\partial \Omega$,

$$
\begin{gathered}
\nabla \cdot u=0 \quad \text { in } \Omega \times(0, T) \\
u=0 \text { on } \partial \Omega \times[0, T] \\
u(x, 0)=u_{0}(x) \quad \text { in } \Omega .
\end{gathered}
$$

Here, $\Omega$ is a bounded domain in $R^{3}$ with smooth boundary $\partial \Omega, u(x, t)$ denotes the velocity of the fluid and $f(x, t)$ stands for external force. We assume that the density, the yield limit and the viscosity are positive constants. In particular, the

Received by the editors August 15, 1986.

1980 Mathematics Subject Classification (1985 Revision). Primary 35Q20, 35B10, 35B40, 35B65; Secondary 76A05, 76D99.

This research was supported by the Air Force Office of Scientific Research under grant AFOSR86-0085 and by NSF grant DMS-8521848. 
density is taken to be one. We employ the notation

$$
\begin{gathered}
a(u, w)=\sum_{i, j=1}^{3} 2 \mu \int_{\Omega} D_{i j}(u) D_{i j}(w) d x, \quad \mu=\text { viscosity } \\
D_{i j}(u)=\frac{1}{2}\left(\frac{\partial u_{i}}{\partial x_{j}}+\frac{\partial u_{j}}{\partial x_{i}}\right) \\
J(u)=2 g \int_{0} D_{\Pi}(u)^{1 / 2} d x, \quad g=\text { yield limit } \\
D_{\Pi}(u)=\frac{1}{2} \sum_{i, j=1}^{3} D_{i j}(u)^{2} \\
b(u, v, w)=\sum_{i, j=1}^{3} \int_{\Omega} u_{j} \frac{\partial v_{i}}{\partial x_{j}} w_{i} d x \\
\langle f, h\rangle=\sum_{i=1}^{3} \int_{\Omega} f_{i} h_{i} d x
\end{gathered}
$$

Duvaut and Lions [3] gave a detailed derivation of (0-1) and proved the existence of weak solutions of (0-1) to (0-4). They $[2,3]$ also obtained more regular solutions in a two dimensional domain. For a variant of Bingham fluid, Naumann and Wulst [9] established the existence of the same kind of regular solutions in a three dimensional domain through a different method. They [9] assumed that the initial data belong to a special class of stationary states and essentially used the assumption of "averaged nonlinear viscosity." Our model described by (0-1) does not satisfy this assumption and the result of [9] cannot be applied.

In this paper we prove the existence of local solutions of (0-1) to (0-4) which are similarly regular under the same assumptions on the data as in [9]. Since (0-1) reduces to the Navier-Stokes equations when $g=0$, we are tempted to utilize the known techniques of analysis for the Navier-Stokes equations. The main task is to deal with the functional $J(\cdot)$ properly. When the space domain has a boundary, the Laplacian does not commute with the projection operator (onto the divergence free vector fields) and this is a major obstacle to taking advantage of the convexity of $J(\cdot)$ in obtaining the regularity in the space variable. For a domain without boundary, some known results for the Navier-Stokes equations have been shown to be valid for (0-1) (see Kim [7] and Renardy [10]). The method in [7] obviously fails in the present problem for the reason mentioned above. A different idea is to express the regularity in the space variable in terms of the time derivative with the crucial help of the $L^{p}$-theory of the Stokes operator due to Cattabriga [1] and Giga $[\mathbf{5}, \mathbf{6}]$. Then the basic energy inequality used by Duvaut and Lions [3] for a two dimensional domain can be still used for a three dimensional domain to derive useful estimates. For this procedure, we have to analyze a certain class of stationary states in detail and also have to regularize the orginal problem so that the manipulation to get estimates can be justified. Finally, we also obtain global solutions and time-periodic solutions under the assumption of small data. This can be done fairly easily once we establish the basic estimates for the local solution. 
1. Notations and preliminaries. Throughout this paper, $t$ is the time variable, $x=\left(x_{1}, x_{2}, x_{3}\right)$ is the space variable and we employ the notation

$$
\partial_{t}=\frac{\partial}{\partial t}, \quad \partial_{i}=\frac{\partial}{\partial x_{i}}, \quad i=1,2,3 .
$$

For $\alpha=\left(\alpha_{1}, \alpha_{2}, \alpha_{3}\right), \partial^{\alpha}=\partial_{1}^{\alpha_{1}} \partial_{2}^{\alpha_{2}} \partial_{3}^{\alpha_{3}},|\alpha|=\alpha_{1}+\alpha_{2}+\alpha_{3}, \Delta=\sum_{i=1}^{3} \partial_{i}^{2}, \nabla=$ $\left(\partial_{1}, \partial_{2}, \partial_{3}\right)$.

For $v \in\left[L^{2}(\Omega)\right]^{3},\|v\|=\langle v, v\rangle^{1 / 2}$.

When $E$ is a Banach space other than $\left[L^{2}(\Omega)\right]^{3}$, its norm is denoted by $\|\cdot\|_{E}$. We shall use a regularized version of $J(\cdot)$ :

$$
J_{\varepsilon}(v)=2 g \int_{\Omega}\left(\varepsilon+D_{\Pi}(v)\right)^{1 / 2} d x, \quad \varepsilon>0 .
$$

We introduce the function spaces

$$
\begin{gathered}
S=\left\{\phi \in\left[C_{0}^{\infty}(\Omega)\right]^{3}: \nabla \cdot \phi=0\right\}, \\
W^{m, r}(\Omega)=\left\{v \in L^{r}(\Omega): \partial^{\alpha} v \in L^{r}(\Omega), 1 \leq|\alpha| \leq m\right\}, \\
W_{0}^{m, r}(\Omega)=\text { the completion of } C_{0}^{\infty}(\Omega) \text { in } W^{m, r}(\Omega), \\
W^{-m, r^{\prime}}(\Omega)=\text { the dual of } W_{0}^{m, r}, \quad \text { where } \frac{1}{r^{\prime}}+\frac{1}{r}=1,1 \leq r<\infty, \\
X_{r}=\text { completion of } S \text { in }\left[L^{r}(\Omega)\right]^{3} .
\end{gathered}
$$

We let $P_{r}$ denote the projection from $\left[L^{r}(\Omega)\right]^{3}$ onto $X_{r}$ and write the Stokes operator as $A_{r}=-P_{r} \Delta$ with the domain

$$
D\left(A_{r}\right)=\left[W^{2, r}(\Omega)\right]^{3} \cap\left[W_{0}^{1, r}(\Omega)\right]^{3} \cap X_{r} .
$$

When $r=2$, we also write $P=P_{2}, A=A_{2}$. Giga [5] proved that $-A_{r}$ generates a bounded analytic semigroup in $X_{r}, 1<r<\infty$. For $0<\Theta<1$ and $1<r<\infty, A_{r}^{\Theta}$ is well defined and its domain $D\left(A_{r}^{\Theta}\right)$ is equipped with the graph norm. Giga [6] also showed that for $0<\Theta<1,1<r<\infty$,

$$
D\left(A_{r}^{\Theta}\right)=D\left(\left(-\Delta_{r}\right)^{\Theta}\right) \cap X_{r}
$$

where $\Delta_{r}=\Delta$ with the domain

$$
D\left(-\Delta_{r}\right)=\left[W^{2, r}(\Omega)\right]^{3} \cap\left[W_{0}^{1, r}(\Omega)\right]^{3} .
$$

Fujiwara [4] showed that for $1 / 2 r<\Theta \leq 1$,

$$
D\left(\left(-\Delta_{r}\right)^{\Theta}\right)=\left\{v \in\left[\mathcal{H}^{2 \Theta, r}(\Omega)\right]^{3}: v=0 \text { on } \partial \Omega\right\}
$$

where $\mathcal{H}^{2 \Theta, r}(\Omega)$ is the space of restrictions to $\Omega$ of the Bessel potential $\mathcal{H}^{2 \Theta, r}\left(R^{3}\right)$. Since $\mathcal{H}^{1, r}(\Omega)=W^{1, r}(\Omega), 1<r<\infty$,

$$
D\left(A_{r}^{1 / 2}\right)=\left[W_{0}^{1, r}(\Omega)\right]^{3} \cap X_{r} .
$$

As a simple consequence of Theorem 1 of Giga [5], we can derive

LEMMA 1.1. Let $1<r<\infty$ and $0<\Theta<1$. Then, for every $v \in D\left(A_{r}^{\Theta}\right)$ and $\lambda>0$,

$$
\left\|\left(\lambda I+A_{r}\right)^{-1} v\right\|_{D\left(A_{r}^{\Theta}\right)} \leq \frac{C}{\lambda}\|v\|_{D\left(A_{r}^{\Theta}\right)},
$$

where $C$ is a positive constant independent of $\lambda$.

In fact, this will be used in the following special version. 
LEMMA 1.2. Suppose that $v$ and $h$ belong to $\left[W_{0}^{1, r}(\Omega)\right]^{3} \cap X_{r}$ and that for some scalar function $p$ and positive constant $\varepsilon$,

$$
v-\varepsilon \Delta v+\nabla p=h
$$

holds in the sense of distribution in $\Omega$. Then we have

$$
\|v\|_{\left[W_{0}^{1, r}(\Omega)\right]^{3}} \leq C\|h\|_{\left[W_{0}^{1, r}(\Omega)\right]^{3}}
$$

for some positive constant $C$ independent of $\varepsilon$ and $h$.

We shall also need a theorem of Cattabriga $[\mathbf{1}]$ in the following form (see Temam $[12])$.

LEMMA 1.3. Let $h \in\left[W^{-1, r}(\Omega)\right]^{3}, 1<r<\infty$. Then, there are unique functions $v$ and $p$ (unique up to a constant) which are solutions of

$$
\begin{gathered}
-\Delta v+\nabla p=h \quad \text { in } \Omega, \\
\nabla \cdot v=0 \quad \text { in } \Omega, \\
v=0 \quad \text { on } \partial \Omega,
\end{gathered}
$$

such that $v \in\left[W_{0}^{1, r}(\Omega)\right]^{3}, p \in L^{r}(\Omega)$ and

$$
\|v\|_{\left[W_{0}^{1, r}(\Omega)\right]^{3}} \leq C\|h\|_{\left(W^{-1, r}(\Omega)\right]^{3}} .
$$

We shall employ the eigenfunctions of $A$;

$$
A \varphi_{n}=\lambda_{n} \varphi_{n} \quad \text { in } X_{2},
$$

where $0<\lambda_{1} \leq \lambda_{2} \leq \cdots, \lambda_{n} \rightarrow \infty$ as $n \rightarrow \infty$, and

$$
\left\langle\varphi_{n}, \varphi_{m}\right\rangle=\delta_{n m}
$$

Using these eigenfunctions, we define for $s \in R$,

$$
V_{s}=\left\{v=\sum_{n=1}^{\infty} a_{n} \varphi_{n}: a_{n} \in R, \sum_{n=1}^{\infty} a_{n}^{2} \lambda_{n}^{s}<\infty\right\}
$$

equipped with the norm

$$
\|v\|_{V_{s}}=\left(\sum_{n=1}^{\infty} a_{n}^{2} \lambda_{n}^{s}\right)^{1 / 2}
$$

It is known that $V_{0}=X_{2}, V_{1}=\left[W_{0}^{1,2}(\Omega)\right]^{3} \cap X_{2}, V_{2}=\left[W^{2,2}(\Omega)\right]^{3} \cap\left[W_{0}^{1,2}(\Omega)\right]^{3} \cap$ $X_{2}, V_{4}=\left\{v \in V_{2}: A v \in V_{2}\right\}$, and, for each $s>0, V_{s} \subset V_{0} \subset V_{-s}$ and $\left[V_{s}, V_{-s}\right]_{1 / 2}=V_{0}$. by

Finally we list some properties of $J_{\varepsilon}(\cdot)$. The Gateaux differential of $J_{\varepsilon}(\cdot)$ is given

$$
\left(J_{\varepsilon}^{\prime}(u), w\right)=g \int_{\Omega} \sum_{i, j=1}^{3}\left(\varepsilon+D_{\Pi}(u)\right)^{-1 / 2} D_{i j}(u) D_{i j}(w) d x
$$

for each $u, w \in\left[W_{0}^{1,2}(\Omega)\right]^{3}$, where $($,$) is the duality pairing between \left[W_{0}^{1,2}(\Omega)\right]^{3}$ and $\left[W^{-1,2}(\Omega)\right]^{3}$. This can also be interpreted as the duality pairing between $V_{1}$ and $V_{-1}$ when $u$ and $w$ belong to $V_{1}$. It is easily seen that $J_{\varepsilon}^{\prime}(\cdot)$ is hemicontinuous 
and bounded as a mapping from $\left[W_{0}^{1,2}(\Omega)\right]^{3}$ into $\left[W^{-1,2}(\Omega)\right]^{3}$ and also as a mapping from $V_{1}$ into $V_{-1}$. Since $J_{\varepsilon}(\cdot)$ is convex, $J_{\varepsilon}^{\prime}(\cdot)$ is monotone and consequently, for every $u \in L^{2}\left(0, T ;\left[W_{0}^{1,2}(\Omega)\right]^{3}\right)$ such that $\partial_{t} u \in L^{2}\left(0, T ;\left[W_{0}^{1,2}(\Omega)\right]^{3}\right)$,

$$
\left(\partial_{t} J_{\varepsilon}^{\prime}(u), \partial_{t} u\right) \geq 0
$$

holds for almost all $t \in[0, T]$.

2. Stationary states. We shall consider a special class of stationary states of $(0-1),(0-2)$ and (0-3): $\mathcal{G}$ is the set of all $v \in V_{1}$ such that for some $h \in\left[L^{2}(\Omega)\right]^{3}$,

$$
a(v, w-v)+b(v, v, w)+J(w)-J(v) \geq\langle h, w-v\rangle
$$

holds for every $w \in V_{1}$.

Proposition 2.1. $\mathcal{G} \subset\left[W_{0}^{1,6}(\Omega)\right]^{3}$ and for each $v \in \mathcal{G}$,

$$
\|v\|_{\left[W_{0}^{1,6}(\Omega)\right]^{3}} \leq C\|h\|^{4}+C
$$

where $h$ is a function corresponding to $v$ in (2-1) and $C$ denotes positive constants independent of $h$.

Proof. Choose any $v \in \mathcal{G}$ and let $h$ correspond to $v$ in (2-1). Setting $w \equiv 0$ in (2-1), we obtain

$$
\sum_{i=1}^{3}\left\|\partial_{i} v\right\|^{2} \leq C\|h\|^{2}
$$

where $C$ is a positive constant independent of $h$. Next we define $q=h-\sum_{j=1}^{3} v_{j} \partial_{j} v$. Then, $q \in\left[W^{-1,3}(\Omega)\right]^{3}$ since $L^{2}(\Omega) \subset W^{-1,3}(\Omega)$ and $W^{1,2}(\Omega) \subset L^{6}(\Omega)$. Furthermore, by (2-3),

$$
\|q\|_{\left[W^{-1,3}(\Omega)\right]^{3}} \leq C\left(\|h\|+\|h\|^{2}\right)
$$

for some positive constant $C$. We also define the operator $\bigwedge_{\varepsilon}$ from $V_{1}$ into $V_{-1}$ such that for each $u, w \in V_{1}$,

$$
\left(\bigwedge_{\varepsilon} u, w\right)=a(u, w)+\left(J_{\varepsilon}^{\prime}(u), w\right)
$$

where $($,$) is the duality pairing between V_{1}$ and $V_{-1}$. It is easy to see that for $u, w \in V_{1}$,

$$
a(u, w)=\mu \sum_{j=1}^{3}\left\langle\partial_{j} u, \partial_{j} w\right\rangle .
$$

Since $J_{\varepsilon}^{\prime}(\cdot)$ is monotone, bounded and hemicontinuous, so is $\bigwedge_{\xi}$. Moreover,

$$
\frac{\left(\bigwedge_{\varepsilon} u, u\right)}{\|u\|_{V_{1}}} \rightarrow \infty \quad \text { as }\|u\|_{V_{1}} \rightarrow \infty
$$

Hence, according to Theorem 2.1 of Lions [8], there is a functions $v_{\varepsilon} \in V_{1}$, such that

$$
\left(\bigwedge_{\varepsilon} v_{\varepsilon}, w\right)=\left(i^{*} q, w\right) \text { for all } w \in V_{1}
$$


where $i^{*}$ is the adjoint of the continuous embedding $i$ from $V_{1}$ into $\left[W_{0}^{1,3 / 2}(\Omega)\right]^{3}$. Now (2-7) implies that for some scalar function $p_{\varepsilon}$,

$$
-\mu \Delta v_{\varepsilon}-g \sum_{j=1}^{3} \partial_{j}\left\{\left(\varepsilon+D_{\Pi}\left(v_{\varepsilon}\right)\right)^{-1 / 2} D_{i j}\left(v_{\varepsilon}\right)\right\}+\nabla p_{\varepsilon}=q
$$

holds in the sense of distribution in $\Omega$. The second term represents a $R^{3}$-valued function in terms of its $i$ th component. By virtue of

$$
\left\|\left(\varepsilon+D_{\Pi}(w)\right)^{-1 / 2} D_{i j}(w)\right\|_{L^{\infty}(\Omega)} \leq \sqrt{2}, \quad i, j=1,2,3 \text {, for all } w \in\left[W_{0}^{1,2}(\Omega)\right]^{3},
$$

we can use Lemma 1.3 to derive

$$
\left\|v_{\varepsilon}\right\|_{\left[W_{0}^{1,3}(\Omega)\right]^{3}} \leq C\|q\|_{\left[W^{-1,3}(\Omega)\right]^{3}}+C
$$

where $C$ denotes positive constants independent of $\varepsilon$. In the meantime, (2-7) also implies that for every $w \in V_{1}$,

$$
a\left(v_{\varepsilon}, w-v_{\varepsilon}\right)+J_{\varepsilon}(w)-J_{\varepsilon}\left(v_{\varepsilon}\right) \geq\left(i^{*} q, w-v_{\varepsilon}\right) .
$$

We can extract a subsequence still denoted by $\left\{v_{\varepsilon}\right\}$ such that $v_{\varepsilon} \rightarrow u$ weakly in $V_{1}$ and $\left[W_{0}^{1,3}(\Omega)\right]^{3}$ for some $u \in V_{1} \cap\left[W_{0}^{1,3}(\Omega)\right]^{3}$, which also satisfies

$$
a(u, w-u)+J(w)-J(u) \geq\left(i^{*} q, w-u\right)
$$

for every $w \in V_{1}$. By the uniqueness of solution of (2-12), $u \equiv v$ and, by (2-4) and $(2-10)$

$$
\|v\|_{\left[W_{0}^{1,3}(\Omega)\right]^{3}} \leq C\left\{\|h\|+\|h\|^{2}\right\}+C
$$

where $C$ denotes positive constants. Now we find that $q \in\left[W^{-1,6}(\Omega)\right]^{3}$ since $L^{2}(\Omega) \subset W^{-1,6}(\Omega)$ and $W_{0}^{1,3}(\Omega) \subset L^{r}(\Omega)$, for any $1 \leq r<\infty$.

Furthermore, (2-13) yields

$$
\|Q\|_{\left[W^{-1,6}(\Omega)\right]^{3}} \leq C\|h\|^{4}+C
$$

where $C$ denotes positive constants. With the aid of (2-9), we can repeat the above argument to arrive at $v \in\left[W_{0}^{1,6}(\Omega)\right]^{3}$ and (2-2).

REMARK 2.2. Even if $v \in S, v$ may not belong to $\mathcal{G}$. An example in a two dimensional domain was given in [7].

PROPOSITION 2.3. $g$ is dense in $V_{1}$.

ProOF. Since $J(\cdot)$ is a continuous convex functional in $\left[W_{0}^{1,2}(\Omega)\right]^{3}$, its subdifferential $\partial J(u)$ is not empty for each $u \in\left[W_{0}^{1,2}(\Omega)\right]^{3}$. Choose any $u \in V_{1}$ and $\delta>0$. Then, there is $\psi \in \partial J(u)$ in $\left[W^{-1,2}(\Omega)\right]^{3}$ such that

$$
J(w)-J(u) \geq(\psi, w-u)
$$

for every $w \in\left[W_{0}^{1,2}(\Omega)\right]^{3}$ where $($,$) is the duality pairing between \left[W_{0}^{1,2}(\Omega)\right]^{3}$ and $\left[W^{-1,2}(\Omega)\right]^{3}$. Thus

$$
\mu \sum_{i=1}^{3}\left\langle\partial_{i} u, \partial_{i} w-\partial_{i} u\right\rangle+J(w)-J(u) \geq(q, w-u)
$$


holds for every $w \in\left[W_{0}^{1,2}(\Omega)\right]^{3}$ where $q=\psi-\mu \Delta u \in\left[W^{-1,2}(\Omega)\right]^{3} \subset V_{-1}$. In particular, (2-16) holds for every $w \in V_{1}$ and ( , ) can be interpreted as the duality pairing between $V_{1}$ and $V_{-1}$. Now we can find $q^{*} \in\left[L^{2}(\Omega)\right]^{3}$ such that

$$
\left\|q-q^{*}\right\|_{\left[W^{-1,2}(\Omega)\right]^{3}}<\delta .
$$

By the same argument as in the proof of Proposition 2.1, it can be shown that there is $v \in V_{1} \cap\left[W_{0}^{1,6}(\Omega)\right]^{3}$ satisfying

$$
\mu \sum_{i=1}^{3}\left\langle\partial_{i} v, \partial_{i} w-\partial_{i} v\right\rangle+J(w)-J(v) \geq\left\langle q^{*}, w-v\right\rangle
$$

for all $w \in V_{1}$. Now it follows from (2-16), (2-17) and (2-18) that

$$
\|u-v\|_{V_{1}} \leq C\left\|q-q^{*}\right\|_{\left[W^{-1,2}(\Omega)\right]^{3}}<C \delta
$$

for some positive constant $C$ independent of $\delta$. Since $\sum_{j=1}^{3} v_{j} \partial_{j} v \in\left[L^{2}(\Omega)\right]^{3}, v$ satisfies (2-1) with $h=q^{*}+\sum_{j=1}^{3} v_{j} \partial_{j} v \in\left[L^{2}(\Omega)\right]^{3}$.

PROPOSITION 2.4. Let $v \in \mathcal{G}$ be given with the corresponding $h \in\left[L^{2}(\Omega)\right]^{3}$. Then, for each $\varepsilon>0$, there are $v_{\varepsilon} \in V_{4}$ and $h_{\varepsilon} \in\left[L^{2}(\Omega)\right]^{3}$ such that

$$
\begin{aligned}
\mu A v_{\varepsilon} & +\mu \varepsilon A^{2} v_{\varepsilon}-P g \sum_{j=1}^{3} \partial_{j}\left\{\left(\varepsilon+D_{\Pi}\left(v_{\varepsilon}\right)\right)^{-1 / 2} D_{i j}\left(v_{\varepsilon}\right)\right\} \\
& +P \sum_{j=1}^{3} v_{\varepsilon j} \partial_{j} v_{\varepsilon}=P h_{\varepsilon}
\end{aligned}
$$

holds in $V_{0}$ and such that $v_{\varepsilon} \rightarrow v$ weakly in $V_{1}, h_{\varepsilon} \rightarrow h$ weakly in $\left[L^{2}(\Omega)\right]^{3}$ as $\varepsilon \rightarrow 0$ and

$$
\left\|h_{\varepsilon}\right\| \leq C\left(\|h\|+\|h\|^{2}\right)\left(\|h\|^{4}+C\right)
$$

where $C$ denotes positive constants independent of $\varepsilon$ and $h$.

(In the third term of (2-20), the projection operator is applied to a $R^{3}$-valued function which is expressed in terms of its $i$ th component.)

ProOF. Define $q=h-\sum_{j=1}^{3} v_{j} \partial_{j} v$. Then, $q \in\left[L^{2}(\Omega)\right]^{3}$ by Proposition 2.1. We then consider a mapping $\bigwedge_{\varepsilon}$ from $V_{2}$ into $V_{-2}$ such that for each $u, w \in V_{2}$

$$
\begin{aligned}
\left(\bigwedge_{\varepsilon} u, w\right)= & \mu \sum_{j=1}^{3}\left\langle\partial_{j} u, \partial_{j} w\right\rangle+\mu \varepsilon\langle A u, A w\rangle \\
& +g \sum_{i, j=1}^{3} \int\left(\varepsilon+D_{\Pi}(u)\right)^{-1 / 2} D_{i j}(u) D_{i j}(w) d x
\end{aligned}
$$

where $\left(\right.$, ) is the duality pairing between $V_{2}$ and $V_{-2}$. As in the proof of Proposition $2.1, \bigwedge_{\varepsilon}$ is hemicontinuous, monotone and bounded such that

$$
\frac{\left(\bigwedge_{\varepsilon} u, u\right)}{\|u\|_{V_{2}}} \rightarrow \infty \quad \text { as }\|u\|_{V_{2}} \rightarrow \infty
$$


Thus, there is $v_{\varepsilon} \in V_{2}$ such that

$$
\left(\bigwedge_{\varepsilon} v_{\varepsilon}, w\right)=\langle q, w\rangle
$$

for every $w \in V_{2}$. It follows from (2-22) that

$$
\mu \varepsilon\left\langle A v_{\varepsilon}, A \phi_{n}\right\rangle=\left\langle\Phi_{\varepsilon}, \phi_{n}\right\rangle \text { for each } n,
$$

where

$$
\Phi_{\varepsilon}=\mu \Delta v_{\varepsilon}+g \sum_{j=1}^{3} \partial_{j}\left\{\left(\varepsilon+D_{\Pi}\left(v_{\varepsilon}\right)\right)^{-1 / 2} D_{i j}\left(v_{\varepsilon}\right)\right\}+q \in\left[L^{2}(\Omega)\right]^{3} .
$$

If $a_{n}=\left\langle v_{\varepsilon}, \phi_{n}\right\rangle$ and $b_{n}=\left\langle\Phi_{\varepsilon}, \phi_{n}\right\rangle,(2-25)$ yields

$$
\sum_{n=1}^{\infty} a_{n}^{2} \lambda_{n}^{4}<\infty
$$

since $\sum_{n=1}^{\infty} b_{n}^{2}<\infty$. Hence $v_{\varepsilon} \in V_{4}$, which implies $A v_{\varepsilon} \in V_{2}$. In the meantime, we derive from $(2.24)$

$$
\begin{aligned}
\left\|v_{\varepsilon}\right\|_{V_{1}} & \leq C\|q\|_{\left[W^{-1,2}(\Omega)\right]^{3}} \leq C\|q\|_{\left[W^{-1,3}(\Omega)\right]^{3}} \\
& \leq C\left(\|h\|+\|h\|^{2}\right), \quad \text { by }(2-4),
\end{aligned}
$$

where $C$ stands for positive constants independent of $\varepsilon$. It also follows from (2-24) that there is a scalar function $p_{\varepsilon}$ such that

$$
-\mu \Delta\left(v_{\varepsilon}+\varepsilon A v_{\varepsilon}\right)-g \sum_{j=1}^{3} \partial_{j}\left\{\left(\varepsilon+D_{\Pi}\left(v_{\varepsilon}\right)\right)^{-1 / 2} D_{i j}\left(v_{\varepsilon}\right)\right\}+\nabla p_{\varepsilon}=q
$$

holds in the sense of distribution in $\Omega$. Let us write $G_{\varepsilon}=v_{\varepsilon}+\varepsilon A v_{\varepsilon}$. Then $G_{\varepsilon} \in V_{2}$ and, by Lemma 1.3 ,

$$
\begin{aligned}
\left\|G_{\varepsilon}\right\|_{\left[W_{0}^{1,6}(\Omega)\right]^{3}} & \leq C\|q\|_{\left[W^{-1,6}(\Omega)\right]^{3}}+C \\
& \leq C\|h\|^{4}+C,
\end{aligned}
$$

where (2-9) and (2-14) have been used and $C$ denotes positive constants independent of $\varepsilon$. Now, for some scalar function $\tilde{p}_{\varepsilon}$,

$$
v_{\varepsilon}-\varepsilon \Delta v_{\varepsilon}+\nabla \tilde{p}_{\varepsilon}=G_{\varepsilon}
$$

holds in the sense of distribution in $\Omega$. With the aid of (1-7) and (2-29), we derive

$$
\left\|v_{\varepsilon}\right\|_{\left[W_{0}^{1,6}(\Omega)\right]^{3}} \leq C\left\|G_{\varepsilon}\right\|_{\left[W_{0}^{1,6}(\Omega)\right]^{3}} \leq C\|h\|^{4}+C,
$$

where $C$ denotes positive constants independent of $\varepsilon$. It follows from (2-27) and (2-31) that

$$
\left\|\sum_{j=1}^{3} v_{\varepsilon j} \partial_{j} v_{\varepsilon}\right\| \leq C\left(\|h\|+\|h\|^{2}\right)\left(C\|h\|^{4}+C\right) \leq C\left(\|h\|+\|h\|^{2}\right)\left(\|h\|^{4}+C\right),
$$

where $C$ denotes positive constants independent of $\varepsilon$. Meanwhile, we can extract a subsequence such that $v_{\varepsilon_{k}} \rightarrow u$ weakly in $V_{1}$ and strongly in $\left[L^{2}(\Omega)\right]^{3}$ as $\varepsilon_{k} \rightarrow 0$ for some function $u \in V_{1}$, which satisfies

$$
\mu \sum_{j=1}^{3}\left\langle\partial_{j} u, \partial_{j} w-\partial_{j} u\right\rangle+J(w)-J(u) \geq\langle q, w-u\rangle
$$


for every $w \in V_{1}$. By the uniqueness of solution of (2-33), $u \equiv v$. Consequently, $v_{\varepsilon} \rightarrow v$ weakly in $V_{1}$ and strongly in $\left[L^{2}(\Omega)\right]^{3}$ as $\varepsilon \rightarrow 0$. We next write

$$
h_{\varepsilon}=q+\sum_{j=1}^{3} v_{\varepsilon j} \partial_{j} v_{\varepsilon} .
$$

Then it is evident that $h_{\varepsilon} \rightarrow h$ weakly in $\left[L^{2}(\Omega)\right]^{3}$ and (2-21) holds.

3. Local existence. Our definition of solution is

DEFINITION 3.1. A function $u(x, t)$ is called a solution of $(0-1)$ to $(0-4)$ if for some $T>0, u \in L^{2}\left(0, T ; V_{1}\right), \partial_{t} u \in L^{2}\left(0, T ; V_{-1}\right), u(x, 0)=u_{0}(x)$ and for almost all $t \in[0, T],(0-1)$ is satisfied for all $w \in V_{1}$.

The main result is

THEOREM 3.2. If

$$
u_{0}(x) \in \mathcal{G}, \quad f \in C\left([0, T] ;\left[L^{2}(\Omega)\right]^{3}\right) \quad \text { and } \quad \partial_{t} f \in L^{2}\left(0, T ;\left[W^{-1,2}(\Omega)\right]^{3}\right),
$$

then there is a unique solution $u(x, t)$ of (0-1) to (0-4) on an interval $\left[0, T^{*}\right], 0<$ $T^{*} \leq T$. Furthermore,

$$
\begin{gathered}
u \in L^{\infty}\left(0, T^{*} ; V_{1} \cap\left[W_{0}^{1,6}(\Omega)\right]^{3}\right), \\
\partial_{t} u \in L^{2}\left(0, T^{*} ; V_{1}\right) \cap L^{\infty}\left(0, T^{*} ; V_{0}\right) .
\end{gathered}
$$

We shall outline the strategy of proof. We first set up a regularized problem with parameter $\varepsilon>0$ associated with (0-1) to (0-4), and obtain solutions which are so regular that the manipulation to obtain energy estimates can be justified. We then obtain sufficient energy estimates independent of $\varepsilon>0$, for which the results of Cattabriga [1] and Giga [5] are crucially used. Finally we pass $\varepsilon \rightarrow 0$ so that the limit provides a solution of (0-1) to (0-4).

3.1. Regularized problem. As above, we suppose that

$$
u_{0}(x) \in \mathcal{G}, \quad f \in C\left([0, T] ;\left[L^{2}(\Omega)\right]^{3}\right) \quad \text { and } \quad \partial_{t} f \in L^{2}\left(0, T ;\left[W^{-1,2}(\Omega)\right]^{3}\right) .
$$

Using $u_{0}(x)$ and its corresponding function $h(x)$, we can construct $u_{0 \varepsilon}(x)$ and $h_{\varepsilon}(x)$ for each $\varepsilon>0$ according to Proposition 2.4. The assertion for the regularized problem is

Proposition 3.3. For each $\varepsilon>0$, there is a scalar function $p_{\varepsilon}(x, t)$ and a unique function $u_{\varepsilon}(x, t)$ such that

$$
\begin{aligned}
\partial_{t} u_{\varepsilon}= & \mu \Delta u_{\varepsilon}+\mu \varepsilon \Delta A u_{\varepsilon}+g \sum_{j=1}^{3} \partial_{j}\left\{\left(\varepsilon+D_{\Pi}\left(u_{\varepsilon}\right)\right)^{-1 / 2} D_{i j}\left(u_{\varepsilon}\right)\right\} \\
& -\sum_{j=1}^{3} u_{\varepsilon j} \partial_{j} u_{\varepsilon}+\nabla p_{\varepsilon}+f, \quad \text { in } \Omega \times(0, T)
\end{aligned}
$$

$$
\begin{gathered}
u_{\varepsilon}(x, 0)=u_{0 \varepsilon}(x) \quad \text { in } \Omega, \\
u_{\varepsilon} \in L^{2}\left(0, T ; V_{4}\right) \cap C\left([0, T] ; V_{3}\right), \\
\partial_{t} u_{\varepsilon} \in L^{2}\left(0, T ; V_{2}\right) \cap C\left([0, T] ; V_{0}\right) .
\end{gathered}
$$


PrOOF. Fix any $\varepsilon>0$. Let us write by using the eigenfunctions in (1-12),

$$
u_{m}(x, t)=\sum_{k=1}^{m} a_{m k}(t) \varphi_{k}(x)
$$

and consider

$$
\begin{gathered}
\left\langle\partial_{t} u_{m}, \varphi_{k}\right\rangle+\mu\left\langle A u_{m}, \varphi_{k}\right\rangle+\mu \varepsilon\left\langle A^{2} u_{m}, \varphi_{k}\right\rangle \\
+b\left(u_{m}, u_{m}, \varphi_{k}\right)+\left(J_{\varepsilon}^{\prime}\left(u_{m}\right), \varphi_{k}\right)=\left\langle f, \varphi_{k}\right\rangle, \quad k=1, \ldots, m, \\
u_{m}(x, 0)=\sum_{k=1}^{m}\left\langle u_{0 \varepsilon}(x), \varphi_{k}\right\rangle \varphi_{k} .
\end{gathered}
$$

We can find $a_{m k}(t) \in C^{1}\left(\left[0, T_{m}\right]\right)$ such that $\partial_{t}^{2} a_{m k} \in L^{2}\left(0, T_{m}\right), k=1, \ldots, m$, for some $0<T_{m} \leq T$ as solutions of (3-8) and (3-9). By virtue of the inequality

$$
\begin{gathered}
\frac{1}{2} \frac{d}{d t}\left\|u_{m}\right\|^{2}+\mu \sum_{j=1}^{3}\left\|\partial_{j} u_{m}\right\|^{2}+\mu \varepsilon\left\|A u_{m}\right\|^{2} \\
\leq\|f\|\left\|u_{m}\right\| \text { for all } t \in\left[0, T_{m}\right],
\end{gathered}
$$

which follows from (3-8), we can set $T_{m}=T$ and derive, by (3-9) and (2-27),

$$
\begin{gathered}
\left\|u_{m}\right\| \leq M \quad \text { for all } t \in[0, T], \\
\sum_{j=1}^{3} \int_{0}^{T}\left\|\partial_{j} u_{m}\right\|^{2} d t \leq M, \\
\int_{0}^{T}\left\|A u_{m}\right\|^{2} d t \leq M_{\varepsilon} .
\end{gathered}
$$

Here and below, $M$ and $M_{\varepsilon}$ denote positive constants independent of $m$, and $M$ is also independent of $\varepsilon$. Next we substitute $A u_{m}$ for $\varphi_{k}$ in (3-8):

$$
\begin{aligned}
& \frac{1}{2} \frac{d}{d t} \sum_{j=1}^{3}\left\|\partial_{j} u_{m}\right\|^{2}+\mu\left\|A u_{m}\right\|^{2}+\mu \varepsilon \sum_{j=1}^{3}\left\|\partial_{j} A u_{m}\right\|^{2} \\
& \quad \leq\left|b\left(u_{m}, u_{m}, A u_{m}\right)\right|+\left|\left(J_{\varepsilon}^{\prime}\left(u_{m}\right), A u_{m}\right)\right|+\left|\left\langle f, A u_{m}\right\rangle\right|
\end{aligned}
$$

for all $t \in[0, T]$. The right-hand side can be estimated as

(3-15) $\left|b\left(u_{m}, u_{m}, A u_{m}\right)\right| \leq M\left\|u_{m}\right\|\left\|A u_{m}\right\| \sum_{j=1}^{3}\left\|\partial_{j} A u_{m}\right\| \quad$ for all $t \in[0, T]$,

$$
\left|\left(J_{\varepsilon}^{\prime}\left(u_{m}\right), A u_{m}\right)\right| \leq M \sum_{j=1}^{3}\left\|\partial_{j} A u_{m}\right\| \quad \text { for all } t \in[0, T], \text { by }(2-9) .
$$

Combining (3-11), (3-13) through (3-16), we obtain

$$
\begin{gathered}
\sum_{j=1}^{3}\left\|\partial_{j} u_{m}\right\| \leq M_{\varepsilon} \quad \text { for all } t \in[0, T], \\
\sum_{j=1}^{3} \int_{0}^{T}\left\|\partial_{j} A u_{m}\right\|^{2} d t \leq M_{\varepsilon} .
\end{gathered}
$$


We then substitute $A^{2} u_{m}$ for $\varphi_{k}$ in (3-8):

(3-19)

$$
\begin{aligned}
& \frac{1}{2} \frac{d}{d t}\left\|A u_{m}\right\|^{2}+\mu \sum_{j=1}^{3}\left\|\partial_{j} A u_{m}\right\|^{2}+\mu \varepsilon\left\|A^{2} u_{m}\right\|^{2} \\
& \quad \leq\left|b\left(u_{m}, u_{m}, A^{2} u_{m}\right)\right|+\left|\left(J_{\varepsilon}^{\prime}\left(u_{m}\right), A^{2} u_{m}\right)\right|+\left|\left\langle f, A^{2} u_{m}\right\rangle\right| \\
& \quad \leq M\left\|A u_{m}\right\|\left\|A^{2} u_{m}\right\| \sum_{j=1}^{3}\left\|\partial_{j} u_{m}\right\|+M_{\varepsilon}\left\|A^{2} u_{m}\right\|\left\|A u_{m}\right\|+\|f\|\left\|A^{2} u_{m}\right\|
\end{aligned}
$$

for all $t \in[0, T]$, which, combined with (3-17), yields

$$
\frac{d}{d t}\left\|A u_{m}\right\|^{2}+2 \mu \sum_{j=1}^{3}\left\|\partial_{j} A u_{m}\right\|^{2}+\mu \varepsilon\left\|A^{2} u_{m}\right\|^{2} \leq M_{\varepsilon}\left\|A u_{m}\right\|^{2}+M_{\varepsilon}\|f\|^{2},
$$

for all $t \in[0, T]$. By virtue of (3-9) and the fact that $u_{0 \varepsilon}(x) \in V_{4}$,

$$
\left\|A u_{m}(x, 0)\right\| \leq M_{\varepsilon} .
$$

Consequently, we infer from (3-13) and (3-20) that

$$
\begin{gathered}
\left\|A u_{m}\right\| \leq M_{\varepsilon} \quad \text { for all } t \in[0, T], \\
\int_{0}^{T}\left\|A^{2} u_{m}\right\|^{2} d t \leq M_{\varepsilon} .
\end{gathered}
$$

We next differentiate (3-8) with respect to $t$ and substitute $\partial_{t} u_{m}$ for $\varphi_{k}$ to derive

$$
\begin{aligned}
& \frac{1}{2} \frac{d}{d t}\left\|\partial_{t} u_{m}\right\|^{2}+\mu \sum_{j=1}^{3}\left\|\partial_{j} \partial_{t} u_{m}\right\|^{2}+\mu \varepsilon\left\|\partial_{t} A u_{m}\right\|^{2} \\
& \quad \leq\left|b\left(\partial_{t} u_{m}, u_{m}, \partial_{t} u_{m}\right)\right|+\left|\left(\partial_{t} f, \partial_{t} u_{m}\right)\right| \\
& \quad \leq M_{\varepsilon}\left\|\partial_{t} u_{m}\right\| \sum_{j=1}^{3}\left\|\partial_{j} \partial_{t} u_{m}\right\|+M\left\|\partial_{t} f\right\|_{\left[W^{-1,2}(\Omega)\right]^{3}} \sum_{j=1}^{3}\left\|\partial_{j} \partial_{t} u_{m}\right\|
\end{aligned}
$$

for almost all $t \in[0, T]$, where (1-17) and (3-22) have been used. Now let us consider (3-8) at $t=0$ after $\varphi_{k}$ is replaced by $\partial_{t} u_{m}(x, 0)$ to estimate $\left\|\partial_{t} u_{m}(x, 0)\right\|$ :

$$
\begin{aligned}
\left\|\partial_{t} u_{m}(x, 0)\right\|^{2} \leq & \mu\left|\left\langle A u_{m}(x, 0), \partial_{t} u_{m}(x, 0)\right\rangle\right|+\mu \varepsilon\left|\left\langle A^{2} u_{m}(x, 0), \partial_{t} u_{m}(x, 0)\right\rangle\right| \\
& +\left|b\left(u_{m}(x, 0), u_{m}(x, 0), \partial_{t} u_{m}(x, 0)\right)\right| \\
& +\left|\left(J_{\varepsilon}^{\prime}\left(u_{m}(x, 0)\right), \partial_{t} u_{m}(x, 0)\right)\right| \\
& +\left|\left\langle f(x, 0), \partial_{t} u_{m}(x, 0)\right\rangle\right| .
\end{aligned}
$$

Again by (3-9) and the fact that $u_{0 \varepsilon}(x) \in V_{4}$, we have

$$
\left\|A^{2} u_{m}(x, 0)\right\| \leq M_{\varepsilon}
$$

and consequently,

$$
\begin{gathered}
\left|b\left(u_{m}(x, 0), u_{m}(x, 0), \partial_{t} u_{m}(x, 0)\right)\right| \leq M_{\varepsilon}\left\|\partial_{t} u_{m}(x, 0)\right\|, \\
\left|\left(J_{\varepsilon}^{\prime}\left(u_{m}(x, 0)\right), \partial_{t} u_{m}(x, 0)\right)\right| \leq M_{\varepsilon}\left\|\partial_{t} u_{m}(x, 0)\right\| .
\end{gathered}
$$


Now it follows from (3-25) that

$$
\left\|\partial_{t} u_{m}(x, 0)\right\| \leq M_{\varepsilon}
$$

which together with (3-24), gives

$$
\begin{gathered}
\left\|\partial_{t} u_{m}\right\| \leq M_{\varepsilon} \quad \text { for all } t \in[0, T], \\
\int_{0}^{T}\left\|\partial_{t} A u_{m}\right\|^{2} d t \leq M_{\varepsilon} .
\end{gathered}
$$

By virtue of (3-23), (3-30) and (3-31), we can extract a subsequence still denoted by $\left\{u_{m}\right\}$ such that for some function $u$, as $m \rightarrow \infty$.

$$
\begin{gathered}
u_{m} \rightarrow u \quad \text { weakly in } L^{2}\left(0, T ; V_{4}\right), \\
\partial_{t} u_{m} \rightarrow \partial_{t} u \quad \text { weakly in } L^{2}\left(0, T ; V_{2}\right), \\
\partial_{t} u_{m} \rightarrow \partial_{t} u \quad \text { weak }^{*} \text { in } L^{\infty}\left(0, T ; V_{0}\right),
\end{gathered}
$$

form which it follows that

$$
u_{m} \rightarrow u \quad \text { strongly in } L^{2}\left(0, T ; V_{3}\right)
$$

and hence, by further extracting subsequence if necessary,

$$
u_{m} \rightarrow u \text { strongly in } V_{3} \text {, for almost all } t \in[0, T] .
$$

Now it is easily seen that for almost all $t \in[0, T]$,

$$
\left(J_{\varepsilon}^{\prime}\left(u_{m}\right), \varphi\right) \rightarrow\left(J_{\varepsilon}^{\prime}(u), \varphi\right)
$$

for all $\varphi \in V_{1}$. For the convergence of other terms in (3-8), we can proceed as in the case of the Navier-Stokes equations (see Temam [12]) to deduce that for almost all $t \in[0, T]$,

$$
\left\langle\partial_{t} u, \varphi\right\rangle+\mu\langle A u, \varphi\rangle+\mu \varepsilon\left\langle A^{2} u, \varphi\right\rangle+b(u, u, \varphi)+\left(J_{\varepsilon}^{\prime}(u), \varphi\right)=\langle f, \varphi\rangle
$$

holds for all $\varphi \in V_{1}$, and

$$
u(x, 0)=u_{0 \varepsilon}(x) .
$$

Now (3-3) follows from (3-38). In the meantime, (3-32) and (3-33) imply $u \in$ $C\left([0, T] ; V_{3}\right)$ possiby after a modification on a set of measure zero on $[0, T]$. We next choose any $\psi \in C_{0}^{\infty}\left((0, T) ; V_{2}\right)$. It follows from (3-38) that

$$
\begin{aligned}
\int_{0}^{T}\left\langle\partial_{t} u, \partial_{t} \psi\right\rangle d t= & -\mu \int_{0}^{T}\left\langle A u, \partial_{t} \psi\right\rangle d t \\
& -\mu \varepsilon \int_{0}^{T}\left\langle A u, \partial_{t} A \psi\right\rangle d t-\int_{0}^{T} b\left(u, u, \partial_{t} \psi\right) d t \\
& -\int_{0}^{T}\left(J_{\varepsilon}^{\prime}(u), \partial_{t} \psi\right) d t+\int_{0}^{T}\left\langle f, \partial_{t} \psi\right\rangle d t
\end{aligned}
$$

Since $\partial_{t} u \in L^{2}\left(0, T ; V_{2}\right) \cap L^{\infty}\left(0, T ; V_{0}\right)$ and $\partial_{t} f \in L^{2}\left(0, T ;\left[W^{-1,2}(\Omega)\right]^{3}\right)$, we have

$$
\left|\int_{0}^{T}\left\langle\partial_{t} u, \partial_{t} \psi\right\rangle d t\right| \leq M_{\varepsilon}\left(\int_{0}^{T}\|\psi\|_{V_{2}}^{2} d t\right)^{1 / 2}
$$


where $M_{\varepsilon}$ is a positive constant independent of $\psi$. Hence, we conclude

$$
\partial_{t}^{2} u \in L^{2}\left(0, T ; V_{-2}\right),
$$

from which it follows that $\partial_{t} u \in C\left([0, T] ; V_{0}\right)$ possibly after a modification on a set of measure zero on $[0, T]$. Under the regularity condition (3-5) and (3-6), one can easily show the uniqueness of $u$ by using the monotonicity of $J_{\varepsilon}^{\prime}(\cdot)$.

3.2. New estimates and the proof of Theorem 3.2. Let $u_{\varepsilon}$ be the solution in Proposition 3.3. We write

$$
\mathcal{F}_{\varepsilon}=u_{\varepsilon}+\varepsilon A u_{\varepsilon}
$$

Then, (3-3) can be written as

$$
\begin{aligned}
\mu \Delta \mathcal{F}_{\varepsilon}+\nabla p_{\varepsilon}= & \partial_{t} u_{\varepsilon}-g \sum_{j=1}^{3} \partial_{j}\left\{\left(\varepsilon+D_{\Pi}\left(u_{\varepsilon}\right)\right)^{-1 / 2} D_{i j}\left(u_{\varepsilon}\right)\right\} \\
& +\sum_{j=1}^{3} u_{\varepsilon j} \partial_{j} u_{\varepsilon}-f \quad \text { in } \Omega \times(0, T) .
\end{aligned}
$$

Since $u_{\varepsilon}$ satisfies (3-5) and (3-6), we find that each term of the right-hand side belongs to $C\left([0, T] ;\left[L^{2}(\Omega)\right]^{3}\right)$ and $\mathcal{F}_{\varepsilon} \in C\left([0, T] ; V_{1}\right)$, which implies

$$
p_{\varepsilon} \in C\left([0, T] ; W^{1,2}(\Omega)\right),
$$

$$
\mathcal{F}_{\varepsilon} \in C\left([0, T] ; V_{2}\right) \text {. }
$$

Frorn (3-44), we can derive

$$
\sum_{j=1}^{3}\left\|\partial_{j} u_{\varepsilon}\right\|^{2} \leq C\left(\left\|\partial_{t} u_{\varepsilon}\right\|^{2}+\|f\|^{2}\right) \quad \text { for all } t \in[0, T] .
$$

Here and below, $C$ denotes positive constants independent of $\varepsilon$. By (3-47), we have

$$
\left\|\sum_{j=1}^{3} u_{\varepsilon j} \partial_{j} u_{\varepsilon}\right\|_{\left[W^{-1,3}(\Omega)\right]^{3}} \leq C\left\|u_{\varepsilon}\right\|_{\left[L^{6}(\Omega)\right]^{3}}^{2} \leq C\left(\left\|\partial_{t} u_{\varepsilon}\right\|^{2}+\|f\|^{2}\right)
$$

for all $t \in[0, T]$. With the aid of Lemma 1.3, we obtain from (3-44)

$$
\left\|\mathcal{F}_{\varepsilon}\right\|_{\left[W_{0}^{1,3}(\Omega)\right]^{3}} \leq C\left(\left\|\partial_{t} u_{\varepsilon}\right\|+\|f\|+\left\|\partial_{t} u_{\varepsilon}\right\|^{2}+\|f\|^{2}\right)+C
$$

for all $t \in[0, T]$. Here, we again used (2-9) and the fact $L^{2}(\Omega) \subset W^{-1,3}(\Omega)$. Now (3-43) implies that

$$
u_{\varepsilon}-\varepsilon \Delta u_{\varepsilon}+\nabla \tilde{p}_{\varepsilon}=\mathcal{F}_{\varepsilon}
$$

holds for some scalar function $\tilde{p}_{\varepsilon}$. Since $u_{\varepsilon}$ and $\mathcal{F}_{\varepsilon}$ belong to $\left[W_{0}^{1,3}(\Omega)\right]^{3} \cap X_{3}$ for each $t \in[0, T]$, we can apply (1-7) to obtain

$$
\begin{aligned}
\left\|u_{\varepsilon}\right\|_{\left[W_{0}^{1,3}(\Omega)\right]^{3}} & \leq C\left\|\mathcal{F}_{\varepsilon}\right\|_{\left[W_{0}^{1,3}(\Omega)\right]^{3}} \\
& \leq C\left(\left\|\partial_{t} u_{\varepsilon}\right\|^{2}+\|f\|^{2}\right)+C \text { for all } t \in[0, T], \text { by }(3-49)
\end{aligned}
$$


Consequently, we also obtain

$$
\begin{aligned}
\left\|\sum_{j=1}^{3} u_{\varepsilon j} \partial_{j} u_{\varepsilon}\right\| & \leq C\left\|u_{\varepsilon}\right\|_{\left[W_{0}^{1,3}(\Omega)\right]^{3}}^{2} \\
& \leq C\left(\left\|\partial_{t} u_{\varepsilon}\right\|^{4}+\|f\|^{4}\right)+C
\end{aligned}
$$

for all $t \in[0, T]$. By using (2-9) and the fact $L^{2}(\Omega) \subset W^{-1,6}(\Omega)$, we can repeat the above argument to arrive at

$$
\left\|u_{\varepsilon}\right\|_{\left[W_{0}^{1,6}(\Omega)\right]^{3}} \leq C\left(\left\|\partial_{t} u_{\varepsilon}\right\|^{4}+\|f\|^{4}\right)+C
$$

for all $t \in[0, T]$. Recalling that $\partial_{t}^{2} u_{\varepsilon} \in L^{2}\left(0, T ; V_{-2}\right)$ and $\partial_{t} u_{\varepsilon} \in L^{2}\left(0, T ; V_{2}\right)$, we can borrow Lemma 1.2 of Temam [12, p. 260] to assert

$$
\frac{1}{2} \frac{d}{d t}\left\|\partial_{t} u_{\varepsilon}\right\|^{2}=\left(\partial_{t}^{2} u_{\varepsilon}, \partial_{t} u_{\varepsilon}\right)
$$

in the sense of distribution in $(0, T)$, where $($,$) is the duality pairing between$ $V_{2}$ and $V_{-2}$. Since the right-hand side belongs to $L^{1}(0, T),\left\|\partial_{t} u_{\varepsilon}\right\|^{2}$ is absolutely continuous on $[0, T]$. We now combine (3-44) and (3-54) to deduce

$$
\begin{aligned}
& \frac{1}{2} \frac{d}{d t}\left\|\partial_{t} u_{\varepsilon}\right\|^{2}+\mu \sum_{j=1}^{3}\left\|\partial_{j} \partial_{t} u_{\varepsilon}\right\|^{2} \\
& \quad \leq C\left\|\partial_{t} f\right\|_{\left[W^{-1,2}(\Omega)\right]^{3}} \sum_{j=1}^{3}\left\|\partial_{j} \partial_{t} u_{\varepsilon}\right\|+\left|b\left(\partial_{t} u_{\varepsilon}, u_{\varepsilon}, \partial_{t} u_{\varepsilon}\right)\right|
\end{aligned}
$$

for almost all $t \in[0, T]$.

By virtue of (3-53), we have

$$
\begin{aligned}
\left|b\left(\partial_{t} u_{\varepsilon}, u_{\varepsilon}, \partial_{t} u_{\varepsilon}\right)\right| & \leq C\left\|\partial_{t} u_{\varepsilon}\right\|\left\|u_{\varepsilon}\right\|_{\left[L^{\infty}(\Omega)\right]^{3}} \sum_{j=1}^{3}\left\|\partial_{j} \partial_{t} u_{\varepsilon}\right\| \\
& \leq C\left\|\partial_{t} u_{\varepsilon}\right\|\left\|u_{\varepsilon}\right\|_{\left[W_{0}^{1,6}(\Omega)\right]^{3}} \sum_{j=1}^{3}\left\|\partial_{j} \partial_{t} u_{\varepsilon}\right\| \\
& \leq \frac{\mu}{4} \sum_{j=1}^{3}\left\|\partial_{j} \partial_{t} u_{\varepsilon}\right\|^{2}+C\left\|\partial_{t} u_{\varepsilon}\right\|^{2}\left(\left\|\partial_{t} u_{\varepsilon}\right\|^{8}+\|f\|^{8}+C\right)
\end{aligned}
$$

for all $t \in[0, T]$. Therefore, (3-55) can be written as

$$
\begin{aligned}
& \frac{d}{d t}\left\|\partial_{t} u_{\varepsilon}\right\|^{2}+\mu \sum_{j=1}^{3}\left\|\partial_{j} \partial_{t} u_{\varepsilon}\right\|^{2} \\
& \quad \leq C\left\|\partial_{t} f\right\|_{\left[W^{-1,2}(\Omega)\right]^{3}}^{2}+C\left\|\partial_{t} u_{\varepsilon}\right\|^{2}\left(\left\|\partial_{t} u_{\varepsilon}\right\|^{8}+\|f\|^{8}+C\right)
\end{aligned}
$$

for almost all $t \in[0, T]$. Next we proceed to estimate $\left\|\partial_{t} u_{\varepsilon}(x, 0)\right\|$ by making use of (3-44). Since each term of (3-44) belongs to $C\left([0, T] ;\left[L^{2}(\Omega)\right]^{3}\right)$ and, in particular, $\partial_{t} u_{\varepsilon} \in C\left([0, T] ; V_{0}\right)$, we have

$$
\left\|\partial_{t} u_{\varepsilon}(x, 0)\right\| \leq\|P f(x, 0)\|+\left\|H_{\varepsilon}\right\|,
$$


where

$$
\begin{aligned}
H_{\varepsilon}= & \mu A u_{0 \varepsilon}+\mu \varepsilon A^{2} u_{0 \varepsilon}-P g \sum_{j=1}^{3} \partial_{j}\left\{\left(\varepsilon+D_{\Pi}\left(u_{0 \varepsilon}\right)\right)^{-1 / 2} D_{i j}\left(u_{0 \varepsilon}\right)\right\} \\
& +P \sum_{j=1}^{3} u_{0 \varepsilon j} \partial_{j} u_{0 \varepsilon} .
\end{aligned}
$$

Since $u_{0 \varepsilon}$ was chosen according to Proposition 2.4, we find

$$
\left\|H_{\varepsilon}\right\| \leq C \quad \text { for all } \varepsilon>0 \text {. }
$$

Let us write $z_{\varepsilon}(t)=\left\|\partial_{t} u_{\varepsilon}\right\|^{2}$ so that (3-57) reduces to

$$
\frac{d}{d t} z_{\varepsilon} \leq \beta(t)+C_{1} z_{\varepsilon}^{5}+C_{2} z_{\varepsilon} \quad \text { for almost all } t \in[0, T]
$$

where $\beta(t) \in L^{1}(0, T)$ and $C_{1}, C_{2}$ are positive constants independent of $\varepsilon$. It is obvious that $z_{\varepsilon}(t)$ is nonnegative and that the right-hand side of (3-60) satisfies the Carathéodory condition. Thus, we can apply Theorem 16.2 of Szarski [11] to conclude that there is $0<T^{*} \leq T$ independent of $\varepsilon$ such that

$$
\left|z_{\varepsilon}(t)\right| \leq C \text { for all } t \in\left[0, T^{*}\right],
$$

which, combined with (3-57), yields

$$
\int_{0}^{T^{*}} \sum_{j=1}^{3}\left\|\partial_{j} \partial_{t} u_{\varepsilon}\right\|^{2} d t \leq C .
$$

It also follows from (3-53) and (3-61)

$$
\left\|u_{\varepsilon}\right\|_{\left[W_{0}^{1,6}(\Omega)\right]^{3}} \leq C \text { for all } t \in\left[0, T^{*}\right] .
$$

Now we can extract a subsequence still denoted by $\left\{u_{\varepsilon}\right\}$ such that for some function $u$, as $\varepsilon \rightarrow 0$,

$$
\begin{gathered}
u_{\varepsilon} \rightarrow u \quad \text { weak }^{*} \text { in } L^{\infty}\left(0, T^{*} ; V_{1} \cap\left[W_{0}^{1,6}(\Omega)\right]^{3}\right) \\
\partial_{t} u_{\varepsilon} \rightarrow \partial_{t} u \quad \text { weak* in } L^{\infty}\left(0, T^{*} ; V_{0}\right) \\
\partial_{t} u_{\varepsilon} \rightarrow \partial_{t} u \quad \text { weakly in } L^{2}\left(0, T^{*} ; V_{1}\right)
\end{gathered}
$$

which implies

$$
u_{\varepsilon} \rightarrow u \quad \text { strongly in } L^{2}\left(0, T^{*} ; V_{0}\right) .
$$

To proceed further, we observe that each $u_{\varepsilon}$ satisfies

$$
\begin{aligned}
\int_{0}^{T^{*}} & \left\langle\partial_{t} u_{\varepsilon}, \psi-u_{\varepsilon}\right\rangle d t+\mu \sum_{j=1}^{3} \int_{0}^{T^{*}}\left\langle\partial_{j} u_{\varepsilon}, \partial_{j} \psi-\partial_{j} u_{\varepsilon}\right\rangle d t \\
& +\varepsilon \mu \int_{0}^{T^{*}}\left\langle u_{\varepsilon}, A^{2} \psi-A^{2} u_{\varepsilon}\right\rangle d t+\sum_{j=1}^{3} \int_{0}^{T^{*}}\left\langle u_{\varepsilon j} \partial_{j} u_{\varepsilon}, \psi\right\rangle d t \\
& +\int_{0}^{T^{*}}\left(J_{\varepsilon}(\psi)-J_{\varepsilon}\left(u_{\varepsilon}\right)\right) d t \geq \int_{0}^{T^{*}}\left\langle f, \psi-u_{\varepsilon}\right\rangle d t
\end{aligned}
$$


for every $\psi \in L^{2}\left(0, T^{*} ; V_{4}\right)$. In the meantime, we note that

$$
\varliminf_{\varepsilon \rightarrow 0} \int_{0}^{T^{*}} J_{\varepsilon}\left(u_{\varepsilon}\right) d t \geq \lim _{\varepsilon \rightarrow 0} \int_{0}^{T^{*}} J\left(u_{\varepsilon}\right) d t \geq \int_{0}^{T^{*}} J(u) d t .
$$

Now we find upon passing $\varepsilon \rightarrow 0$ in (3-68),

$$
\begin{aligned}
\int_{0}^{T^{*}} & \left\langle\partial_{t} u, \psi-u\right\rangle d t+\mu \sum_{j=1}^{3} \int_{0}^{T^{*}}\left\langle\partial_{j} u, \partial_{j} \psi-\partial_{j} u\right\rangle d t \\
& +\sum_{j=1}^{3} \int_{0}^{T^{*}}\left\langle u_{j} \partial_{j} u, \psi\right\rangle d t+\int_{0}^{T^{*}}(J(\psi)-J(u)) d t \geq \int_{0}^{T^{*}}\langle f, \psi-u\rangle d t
\end{aligned}
$$

for every $\psi \in L^{2}\left(0, T^{*} ; V_{4}\right)$. Using the fact that $V_{4}$ is separable and dense in $V_{1}$, we borrow an argument from Duvaut and Lions [3] to conclude that for almost all $t \in\left[0, T^{*}\right]$,

$$
\begin{aligned}
& \left\langle\partial_{t} u, w-u\right\rangle+\mu \sum_{j=1}^{3}\left\langle\partial_{j} u, \partial_{j} w-\partial_{j} u\right\rangle \\
& +\sum_{j=1}^{3}\left\langle u_{j} \partial_{j} u, w\right\rangle+J(w)-J(u) \geq\langle f, w-u\rangle
\end{aligned}
$$

holds for all $w \in V_{1}$. By making use of (3-64), (3-65) and the fact that $u_{\varepsilon}(x, 0)=$ $u_{0 \varepsilon}(x)$ and $u_{0 \varepsilon} \rightarrow u_{0}$ weakly in $V_{1}$, we can easily derive that $u(x, 0)=u_{0}(x)$. Hence $u(x, t)$ is a solution of $(0-1)$ to $(0-4)$. To prove the uniqueness, let $v(x, t)$ be a solution according to Definition 3.1. Then, we have the inequality

$$
\begin{aligned}
& \left\langle\partial_{t}(u-v), u-v\right\rangle+\mu \sum_{j=1}^{3}\left\langle\partial_{j}(u-v), \partial_{j}(u-v)\right\rangle \\
& \quad \leq|b(u-v, u, u-v)| \text { for almost all } t \in\left[0, T^{*}\right],
\end{aligned}
$$

where we have assumed that $\left[0, T^{*}\right]$ is the common interval. But, by virtue of the fact $u \in L^{\infty}\left(0, T^{*} ; V_{1} \cap\left[W_{0}^{1,6}(\Omega)\right]^{3}\right)$, we have

$$
|b(u-v, u, u-v)| \leq C\|u-v\| \sum_{j=1}^{3}\left\|\partial_{j}(u-v)\right\|
$$

for almost all $t \in\left[0, T^{*}\right]$. We now use the Gronwall inequality to derive from (3-72) that $u \equiv v$.

4. Existence of global solutions and time-periodic solutions. Our assertion is

THEOREM 4.1. Suppose that $u_{0}(x) \in \mathcal{G}, f \in C\left([0, \infty) ;\left[L^{2}(\Omega)\right]^{3}\right)$ and $\partial_{t} f \in$ $L^{\infty}\left(0, \infty ;\left[W^{-1,2}(\Omega)\right]^{3}\right)$. Then there is $\delta>0$ such that if

$$
\begin{gathered}
\|f\| \leq \delta \quad \text { for all } t \geq 0 \\
\left\|\partial_{t} f\right\|_{\left[W^{-1,2}(\Omega)\right]^{3}} \leq \delta \quad \text { for almost all } t \geq 0 \\
\|h\| \leq \delta
\end{gathered}
$$


where $h$ is a function corresponding to $u_{0}$ in (2-1). Then there is a unique global solution $u(x, t)$ of (0-1) to (0-4) such that

$$
\begin{gathered}
u \in L^{\infty}\left(0, \infty ; V_{1} \cap\left[W_{0}^{1,6}(\Omega)\right]^{3}\right), \\
\partial_{t} u \in L^{\infty}\left(0, \infty ; V_{0}\right) \cap L_{\mathrm{loc}}^{2}\left([0, \infty) ; V_{1}\right) .
\end{gathered}
$$

THEOREM 4.2. In addition to the above assumptions possibly with smaller $\delta>$ 0 , we also assume that $f$ is $\eta$-periodic in time. Then, there is an $\eta$-periodic solution $u_{\eta}(x, t)$ of $(0-1),(0-2)$ and (0-3) such that

$$
\begin{gathered}
u_{\eta}(x, t+\eta)=u_{\eta}(x, t) \quad \text { for all } t \in(-\infty, \infty), \\
u_{\eta} \in L^{\infty}\left(0, \eta ; V_{1} \cap\left[W_{0}^{1,6}(\Omega)\right]^{3}\right), \\
\partial_{t} u_{\eta} \in L^{\infty}\left(0, \eta ; V_{0}\right) \cap L^{2}\left(0, \eta ; V_{1}\right) .
\end{gathered}
$$

Furthermore, the solution $u(x, t)$ of Theorem 4.1 converges to $u_{\eta}(x, t)$ in the manner

$$
\sup _{s \geq t}\left\|u(x, s)-u_{\eta}(x, s)\right\| \leq M e^{-\lambda t} \quad \text { for all } t \geq 0
$$

where $M$ and $\lambda$ are positive constants.

As a preparatory step, we present

LEMMA 4.3. Suppose $E(t)$ is nonnegative, locally absolutely continuous on $[0, T)$ and

$$
\frac{d}{d t} E+\nu E \leq C_{1} E^{5}+C_{2} \quad \text { for almost all } t \in[0, T)
$$

where $\nu, C_{1}$ and $C_{2}$ are positive constants. Let $0<\xi<\left(\nu / 32 C_{1}\right)^{1 / 4}$. If $C_{2}<\nu \xi / 2$ and $E(0) \leq \xi$, then $E(t)<2 \xi$ for all $t \in[0, T)$.

Proof. Suppose that the assertion is false. Then, there is $t^{*} \in(0, T)$ such that $E(t)<2 \xi$ for all $t \in\left[0, t^{*}\right)$ and $E\left(t^{*}\right)=2 \xi$. Consequently, we find that

$$
E\left(t^{*}\right) \leq \xi e^{-\nu t^{*} / 2}+\xi\left(1-e^{-\nu t^{*} / 2}\right)<2 \xi
$$

which is a contradiction.

PROOF OF THEOREM 4.1. We first note that the solutions in Propostion 3.3 are defined on $[0, \infty)$ under the assumption that $f \in C\left([0, \infty) ;\left[L^{2}(\Omega)\right]^{3}\right)$ and $\partial_{t} f \in L_{\text {loc }}^{2}\left([0, \infty) ;\left[W^{-1,2}(\Omega)\right]^{3}\right)$. To see this, we rewrite $(3-23),(3-30)$ and $(3-31)$ as

$$
\begin{gathered}
\int_{0}^{T}\left\|A^{2} u_{m}\right\|^{2} d t \leq M(\varepsilon, T), \\
\left\|\partial_{t} u_{m}\right\| \leq M(\varepsilon, T) \quad \text { for all } t \in[0, T], \\
\int_{0}^{T}\left\|\partial_{t} A u_{m}\right\|^{2} d t \leq M(\varepsilon, T),
\end{gathered}
$$

where $M(\varepsilon, T)$ denotes positive constants independent of $m$ which are defined for all $0<T<\infty$ and $\varepsilon>0$. On each finite time interval, we obtain a solution by letting $m \rightarrow \infty$. By the uniquenss of solution, we can define a solution on $[0, \infty)$. We next recall a special case of the Nirenberg-Galiardo inequality: for $0 \leq \alpha<1 / 4$,

$$
\|w\|_{L^{\infty}(\Omega)} \leq C\|w\|_{W^{1,6}(\Omega)}^{1-\alpha}\|w\|_{L^{2}(\Omega)}^{\alpha} \quad \text { for all } w \in W^{1,6}(\Omega) .
$$


Let us fix $0<\alpha<1 / 4$ and rewrite (3-56) as $(4-16)$

$$
\begin{aligned}
\left|b\left(\partial_{t} u_{\varepsilon}, u_{\varepsilon}, \partial_{t} u_{\varepsilon}\right)\right| & \leq C\left\|\partial_{t} u_{\varepsilon}\right\|\left\|u_{\varepsilon}\right\|^{\alpha}\left\|u_{\varepsilon}\right\|_{\left[W_{0}^{1,6}(\Omega)\right]^{3}}^{1-\alpha} \sum_{j=1}^{3}\left\|\partial_{j} \partial_{t} u_{\varepsilon}\right\| \\
& \leq \frac{\mu}{4} \sum_{j=1}^{3}\left\|\partial_{j} \partial_{t} u_{\varepsilon}\right\|^{2}+C\left\|u_{\varepsilon}\right\|^{2 \alpha}\left\|\partial_{t} u_{\varepsilon}\right\|^{2}\left(\left\|\partial_{t} u_{\varepsilon}\right\|^{8}+\|f\|^{8}+C\right),
\end{aligned}
$$

where $C$ still stands for positive constants independent of $\varepsilon$. Then, (3-55) can be written as

$$
\begin{aligned}
& \frac{d}{d t}\left\|\partial_{t} u_{\varepsilon}\right\|^{2}+\mu \sum_{j=1}^{3}\left\|\partial_{j} \partial_{t} u_{\varepsilon}\right\|^{2} \\
& \quad \leq C\left\|\partial_{t} f\right\|_{\left[W^{-1,2}(\Omega)\right]^{3}}^{2}+C\left\|u_{\varepsilon}\right\|^{2 \alpha}\left\|\partial_{t} u_{\varepsilon}\right\|^{2}\left(\left\|\partial_{t} u_{\varepsilon}\right\|^{8}+\|f\|^{8}+C\right)
\end{aligned}
$$

for almost all $t \in[0, \infty)$. In the meantime, it follows from (3-44) that

$$
\frac{d}{d t}\left\|u_{\varepsilon}\right\|^{2}+\mu \sum_{j=1}^{3}\left\|\partial_{j} u_{\varepsilon}\right\|^{2} \leq C\|f\|^{2} \quad \text { for all } t \geq 0 \text {. }
$$

Consequently, for all $t \geq 0$,

$$
\left\|u_{\varepsilon}\right\|^{2} \leq\left\|u_{0 \varepsilon}\right\|^{2}+C \sup _{t \geq 0}\|f\|^{2} .
$$

Now let $0<\delta<1$. If $\|h\| \leq \delta$, then (2-21) yields

$$
\left\|h_{\varepsilon}\right\| \leq C \delta
$$

which implies, by (3-58),

$$
\left\|\partial_{t} u_{\varepsilon}(x, 0)\right\| \leq C \delta+\|f(x, 0)\|,
$$

and, by (2-27),

$$
\left\|u_{0 \varepsilon}\right\| \leq C \delta,
$$

where $C$ denotes positive constants independent of $\varepsilon$ and $\delta$. Hence, by assuming (4-1) and (4-2) with $\delta$ which is sufficiently small, but independent of $\varepsilon,(4-17)$ can be put in the framework of Lemma 4.3 so that we arrive at the estimates:

$$
\begin{gathered}
\left\|\partial_{t} u_{\varepsilon}\right\| \leq C \quad \text { for all } t \geq 0 \text { and all } \varepsilon>0 \\
\sum_{j=1}^{3} \int_{0}^{T}\left\|\partial_{j} \partial_{t} u_{\varepsilon}\right\|^{2} d t \leq C(T) \quad \text { for all } T>0 \text { and all } \varepsilon>0
\end{gathered}
$$

where $C$ and $C(T)$ denote positive constants. It then follows from (3-53) and (4-23) that

$$
\left\|u_{\varepsilon}\right\|_{\left[W_{0}^{1,6}(\Omega)\right]^{3}} \leq C \text { for all } t \geq 0 \text { and all } \varepsilon>0 .
$$

Next we choose any finite time interval and find a solution of $(0-1)$ to (0-4) through the same procedure as in the previous section. Finally, we can extend the time interval to $[0, \infty)$ by using the uniqueness of solution. 
PROOF OF THEOREM 4.2. We shall obtain a periodic solution through a well-known procedure. Let $u(x, t)$ be the global solution in Theorem 4.1 and define

$$
v_{k}(x, t)=u(x, t+k \eta), \quad k=1,2, \ldots
$$

Then, $v_{k}$ is a solution of $(0-1),(0-2)$ and $(0-3)$ on the interval $(-k \eta, \infty)$ since $f$ is $\eta$-periodic in $t$. Similarly to (3-72), we have for each $k$

$$
\begin{aligned}
& \frac{1}{2} \frac{d}{d t}\left\|v_{k}-v_{0}\right\|^{2}+\mu \sum_{j=1}^{3}\left\|\partial_{j}\left(v_{k}-v_{0}\right)\right\|^{2} \\
& \quad \leq\left|b\left(v_{k}-v_{0}, v_{0}, v_{k}-v_{0}\right)\right| \leq C\left\|v_{0}\right\|_{\left[L^{\infty}(\Omega)\right]^{3}} \sum_{j=1}^{3}\left\|\partial_{j}\left(v_{k}-v_{0}\right)\right\|^{2} \\
& \quad \leq C\|u\|^{\alpha}\|u\|_{\left[W_{0}^{1,6}(\Omega)\right]^{3}}^{1-\alpha} \sum_{j=1}^{3}\left\|\partial_{j}\left(v_{k}-v_{0}\right)\right\|^{2}
\end{aligned}
$$

for almost all $t \geq 0$, where $0<\alpha<1 / 4$ and $C$ denotes positive constants independent of $k$. By choosing $\delta$ sufficiently small in (4-1), (4-2) and (4-3), (4-27) can reduce to, for each $k$,

$$
\frac{d}{d t}\left\|v_{k}-v_{0}\right\|^{2}+\mu \sum_{j=1}^{3}\left\|\partial_{j}\left(v_{k}-v_{0}\right)\right\|^{2} \leq 0
$$

for almost all $t \geq 0$. Therefore, for each $k$

$$
\left\|v_{k}-v_{0}\right\| \leq M e^{-\tilde{\mu} t} \quad \text { for all } t \geq 0,
$$

where $M=2 \sup _{t \geq 0}\|u(x, t)\|$ and $\tilde{\mu}$ is a positive constant. Now (4-29) yields

$$
\left\|v_{k+k_{1}}-v_{k_{1}}\right\| \leq M e^{-\tilde{\mu} k_{1} \eta} \quad \text { for all } t \geq 0 \text { and all } k .
$$

Hence $\left\{v_{k}\right\}$ is a Cauchy sequence in $C\left([0, \infty) ; V_{0}\right)$ and let $u_{\eta}(x, t)$ stand for its limit. It is apparent that

$$
\sup _{s \geq t}\left\|u_{\eta}(x, s)-u(x, s)\right\| \leq M e^{\tilde{\mu} \eta} e^{-\tilde{\mu} t} \quad \text { for all } t \geq 0,
$$

which proves (4-9). Next we observe that

$$
u_{\eta}(x, t+\eta)=\lim _{k \rightarrow \infty} v_{k}(x, t+\eta)=\lim _{k \rightarrow \infty} v_{k+1}(x, t)=u_{\eta}(x, t),
$$

which shows that $u_{\eta}$ is $\eta$-periodic. Meanwhile, we note that

$$
\begin{gathered}
\left\|v_{k}\right\|_{\left[W_{0}^{1,6}(\Omega)\right]^{3}} \leq M \quad \text { for almost all } t \geq 0, \\
\left\|\partial_{t} v_{k}\right\| \leq M \quad \text { for almost all } t \geq 0,
\end{gathered}
$$$$
\sum_{j=1}^{3} \int_{0}^{T}\left\|\partial_{j} \partial_{t} v_{k}\right\|^{2} d t \leq M(T) \text { for each } 0<T<\infty
$$ 
where $M$ and $M(T)$ stand for positive constants independent of $k$. In fact, (4-33) and (4-34) follow immediately from (4-4) and (4-5). To see (4-35), we need to improve (4-24) by means of (4-17) and (4-23):

$$
\sum_{j=1}^{3} \int_{s}^{s+T}\left\|\partial_{j} \partial_{t} u_{\varepsilon}\right\|^{2} d t \leq C(T) \text { for all } s \geq 0
$$

where $C(T)$ is a positive constant indpendent of $\varepsilon$. It is evident that (4-36) is equally true for $u$, and (4-35) follows. By virtue of (4-33), (4-34) and (4-35), we find that

$$
\begin{gathered}
v_{k} \rightarrow u_{\eta} \quad \text { weak }^{*} \text { in } L^{\infty}\left(0, \infty ; V_{1} \cap\left[W_{0}^{1,6}(\Omega)\right]^{3}\right), \\
\partial_{t} v_{k} \rightarrow \partial_{t} u_{\eta} \text { weak }^{*} \text { in } L^{\infty}\left(0, \infty ; V_{0}\right), \\
\partial_{t} v_{k} \rightarrow \partial_{t} u_{\eta} \quad \text { weakly in } L^{2}\left(0, T ; V_{1}\right) \text { for each } 0<T<\infty,
\end{gathered}
$$

from which (4-6), (4-7) and (4-8) follow. Since each $v_{k}$ is a solution of $(0-1),(0-2)$ and $(0-3)$ in $\Omega \times(0, \infty)$, we can show by an argument similar to that in the previous section that $u_{\eta}$ is also a solution in $\Omega \times(0, \infty)$, in fact in $\Omega \times(-\infty, \infty)$ by extending $u_{\eta}$ to the interval $(-\infty, 0)$ by the $\eta$-periodic condition.

ACKNOWLEDGEMEnT. I would like to thank Professors K. Hannsgen and R. Wheeler for their support during this research.

\section{REFERENCES}

1. L. Cattabriga, Su un problema al contorno relativo al sistema di equazioni di Stokes, Rend. Mat. Sem. Univ. Padova 31 (1961), 308-340.

2. G. Duvaut and J. L. Lions, Écoulement d'un fluide rigide viscoplastique incompressible, C. R. Acad. Sci. Paris 270 (1970), 58-61.

3. _ Inequalities in mechanics and physics, Springer-Verlag, Berlin, Heidelberg and New York, 1976.

4. D. Fujiwara, On the asymptotic behavior of the Green operators for elliptic boundary problems and the pure imaginary powers of some second order operators, J. Math. Soc. Japan 21 (1969), 481-522.

5. Y. Giga, Analyticity of the semigroup generated by the Stokes operator in Lr spaces, Math. Z. 178 (1981), 297-329.

6. Domains of fractional powers of the Stokes operator in Lr spaces, Arch. Rational Mech. Anal. 89 (1985), 251-265.

7. J. Kim, On the Cauchy problem associated with the motion of a Bingham fluid in the plane, Trans. Amer. Math. Soc. 298 (1986), 371-400.

8. J. L. Lions, Quelques méthodes de résolution des problèmes aux limites non linéaires, Dunod and Gauthier-Villars, Paris, 1969.

9. J. Naumann and M. Wulst, On evolution inequalities of Bingham type in three dimension. II, J. Math. Anal. Appl. 70 (1979), 309-325.

10. M. Renardy, Dense imbedding of test functions in certain functions spaces, Trans. Amer. Math. Soc. 298 (1986), 241-243.

11. J. Szarski, Differential inequalities, Polish Scientific Publishers, Warszawa, 1965.

12. R. Temam, Navier-Stokes equations, North-Holland, Amsterdam, New York and Oxford, 1984.

Department of Mathematics, Virginia Polytechnic institute and State UNIVERSITY, BLACKSBURG, VIRGINIA 24061 